University of Nebraska - Lincoln

DigitalCommons@University of Nebraska - Lincoln

Norman R. Simon Papers

Research Papers in Physics and Astronomy

3-1970

Pulsation Analysis For Stars In Thermal Imbalance

Norman R. Simon

University of Nebraska - Lincoln, nsimon@unl.edu

Follow this and additional works at: https://digitalcommons.unl.edu/physicssimon

Simon, Norman R., "Pulsation Analysis For Stars In Thermal Imbalance" (1970). Norman R. Simon Papers. 9.

https://digitalcommons.unl.edu/physicssimon/9

This Article is brought to you for free and open access by the Research Papers in Physics and Astronomy at DigitalCommons@University of Nebraska - Lincoln. It has been accepted for inclusion in Norman R. Simon Papers by an authorized administrator of DigitalCommons@University of Nebraska - Lincoln. 
The Astrophysical Journal, Vol. 159, March 1970

(C) 1970. The University of Chicago. All rights reserved. Printed in U.S.A.

\title{
PULSATION ANALYSIS FOR STARS IN THERMAL IMBALANCE
}

\author{
NoRman R. Simon \\ Institute for Space Studies, Goddard Space Flight Center, NASA, New York, New York \\ Received 1969 June 27; revised 1969 August 25
}

\section{ABSTRACT}

The effects of thermal imbalance $\left(d S_{1} / d t \neq 0\right)$ on stellar pulsational stability have largely been ignored in the literature. Here we have used the linear, quasi-adiabatic pulsation theory to make a preliminary investigation of such effects. Analyses of the standard model and of a white dwarf show that, for these cases, "ordinary" terms in the stability integrals greatly outweigh the "extra" terms considered here. The latter become more important when substantial ionization zones exist in the stellar matter. It is argued that the influence of thermal imbalance on pulsational stability should be quite small for stars crossing the H-R diagram in early post-main-sequence evolution, and for cooling degenerate stars. On the other hand, during pre-main-sequence contraction the thermal-imbalance terms are more likely to be important, and for thermal runaways in shell-burning stars these terms are almost certainly crucial.

\section{INTRODUCTION}

In the familiar linear quasi-adiabatic theory of pulsations, developed in general form by Thomas (1930), the rate of change of the dynamical energy of oscillation $E$ may be written (Ledoux 1958)

$$
\begin{aligned}
\frac{2}{P e r}\left\langle\frac{d E}{d t}\right\rangle & =\int_{0}^{M} \frac{\partial^{2} U}{\partial S \partial \rho} \delta \delta \frac{d S}{d t} d m+\frac{1}{2} \int_{0}^{M} \frac{\partial^{3} U}{\partial S \partial \rho^{2}} \frac{d S_{1}}{d t}(\delta \rho)^{2} d m \\
& +\frac{2}{P e r} \int_{0}^{M}\left\langle\left(\rho-\rho_{1}\right) \frac{d S_{1}}{d t} \frac{\partial^{2} U}{\partial S \partial \rho}\right\rangle d m,
\end{aligned}
$$

where the angular brackets indicate a time average over the pulsation period; the subscript 1 denotes equilibrium quantities; $U$ and $S$ are, respectively, the specific internal energy and entropy of the gas; $\rho$ is the mass density; and the quantity $\delta$ preceding a variable indicates a first-order departure from equilibrium.

In the stellar pulsations most commonly studied, the equilibrium entropy change

$$
\frac{d S_{1}}{d t}=\frac{1}{T}\left[\epsilon-\frac{\partial L_{r}}{\partial m}\right]
$$

where $\epsilon$ is the rate of generation of nuclear energy and $L_{r}$ the luminosity at distance $r$ from the stellar center, is negligible, and the right side of equation (1) reduces to the first integral with

$$
\delta \frac{d S}{d t}=\frac{1}{T}\left[\delta \epsilon-\frac{\partial}{\partial m}\left(\delta L_{r}\right)\right] \text {. }
$$

For those epochs of stellar evolution during which $d S_{1} / d t \neq 0$ (thermal imbalance), the expression (2) must be rewritten as

$$
\delta \frac{d S}{d t}=\frac{1}{T}\left[\delta \epsilon-\frac{\partial}{\partial m}\left(\delta L_{r}\right)\right]-\frac{\delta T}{T^{2}}\left[\epsilon-\frac{\partial L_{r}}{\partial m}\right],
$$

and contributions from the second and perhaps the third integrals on the right side of equation (1) considered. The latter integral was ignored by Thomas as vanishing in the 
mean; however, it was included by Ledoux, who pointed out that second-order contributions from it might subsist.

The purpose of the present work is to consider both the second integral on the right of equation (1) and the extra term introduced in the first integral due to the replacement of equation (2) by equation (3). Because adiabatic evolution $\left(d S_{1} / d t=0\right)$ is so often a good approximation in calculations of stellar models, these terms have, with few exceptions, been ignored in the literature since the general review of Ledoux (1958). In what follows, we shall attempt a preliminary investigation of the problem, proceeding through the use of simple stellar models and semiquantitative estimates to get an idea of the relative size of the thermal-imbalance terms and to draw some conclusions regarding their importance in various phases of stellar evolution.

In \$. II we write equation (1) in more convenient form. Sections III and IV are devoted to evaluating the stability of the standard model and that of a cooling white dwarf, respectively. In $\S \mathrm{V}$ we consider what effects the presence of ionization zones in the stellar matter might have, and in \& VI discuss the results of the various calculations. The final section considers two cases in which thermal-imbalance terms are likely to be important, and for which further investigation should prove fruitful.

II. THE STABILITY INTEGRAL

Equation (1) gives the rate of gain or loss of pulsational energy over a cycle. Let us define the first two integrals on the right-hand side as

$$
L_{P}^{\prime}=\int_{0}^{M} \frac{\partial^{2} U}{\partial S \partial \rho} \delta \rho \delta \frac{d S}{d t} d m+\frac{1}{2} \int_{0}^{M} \frac{\partial^{3} U}{\partial S \partial \rho^{2}} \frac{d S_{1}}{d t}(\delta \rho)^{2} d m .
$$

This represents the contribution to the pulsation analysis of the terms we will consider.

In the usual notation we have

$$
\frac{\partial^{2} U}{\partial S \partial \rho}=\frac{T}{\rho}\left(\Gamma_{3}-1\right)
$$

and by using equation (3), we may rewrite equation (4) as

$$
\begin{gathered}
L_{P}^{\prime}=\int_{0}^{M}\left(\Gamma_{3}-1\right) \frac{\delta \rho}{\rho}[\delta \epsilon \\
\left.-\frac{\partial}{\partial m}\left(\delta L_{r}\right)\right] d m-\int_{0}^{M}\left(\Gamma_{3}-1\right) \frac{\delta \rho}{\rho} \frac{\delta T}{T}\left[\epsilon-\frac{\partial L_{r}}{\partial m}\right] d m \\
+\int_{0}^{M} \frac{\rho^{2}}{2 T} \frac{\partial^{3} U}{\partial S \partial \rho^{2}}\left(\frac{\delta \rho}{\rho}\right)^{2}\left[\epsilon-\frac{\partial L_{r}}{\partial m}\right] d m .
\end{gathered}
$$

Let us now make the assumption that no sources or sinks of subatomic energy exist in the stellar matter. In that event, if the pulsational quantities are defined as

$$
x^{*}=\frac{\delta r}{r}, \quad z^{*}=\frac{\delta \rho}{\rho}, \quad t^{*}=\frac{\delta T}{T}, \quad l^{*}=\frac{\delta L_{r}}{L_{r}},
$$

and with the adiabatic condition

we have

$$
t^{*}=\left(\Gamma_{3}-1\right) z^{*}
$$

$$
\begin{gathered}
\frac{L_{P}^{\prime}}{L}=-\int_{0}^{1}\left(\Gamma_{3}-1\right) z^{*} \frac{1}{L} \frac{\partial}{\partial q}\left(\delta L_{r}\right) d q+\int_{0}^{1}\left(\Gamma_{3}-1\right)^{2} \frac{\partial f}{\partial q}\left(z^{*}\right)^{2} d q \\
-\int_{0}^{1} \frac{\rho^{2}}{2 T} \frac{\partial^{3} U}{\partial S \partial \rho^{2}} \frac{\partial f}{\partial q}\left(z^{*}\right)^{2} d q
\end{gathered}
$$


where $f=L_{r} / L$ and $q=m / M$.

For future convenience we shall rewrite equation (6) as

$$
L^{\prime}{ }_{P} / L=I_{1}+I_{2}+J_{2},
$$

where the integrals (including signs) are labeled in order. We shall call terms coming from the first integral in equation (6) (subscript 1) the "ordinary" terms, while those which arise from the other two integrals (subscript 2) will be referred to as "extra" or "thermal-imbalance" terms.

A star is considered pulsationally stable if $L_{P}^{\prime} / L<0$. In the event that contributions from subatomic energy do exist in the star, one must evidently return to equation (5).

\section{THE STANDARD MODEL}

Following Eddington (1959), let us define the quantity $\eta$ by the relation

$$
f=\eta q \text {. }
$$

Then, if $\kappa$ represents the opacity, the standard model is obtained for

$$
\eta \kappa=\text { const . }
$$

For our analysis, we shall choose the combination

$$
\eta=\text { const }, \quad \kappa=\text { const . }
$$

In that case, since $f=q=1$ at the stellar surface, we must have $\eta=1$, and our choice corresponds to a physically reasonable case of electron-scattering opacity, with luminosity increasing linearly outward with mass fraction.

The standard model is a polytrope of index 3 with a constant ratio $\beta$ of gas pressure to total pressure through the star. The value of $\beta$ depends only on the product $M \mu^{2}$, where $\mu$ is the mean molecular weight of the matter, and may thus be chosen arbitrarily. All of the nonpulsational quantities in equation (6) may be evaluated with the aid of Emden tables (British Association for the Advancement of Science 1932).

The relative pulsational amplitudes $x^{*}, z^{*}$ of the standard model have been given by Schwarzschild (1941) for various values of $\beta$, in both the fundamental and higher modes. We have chosen the lowest of these values of $\beta$ in the fundamental mode as the combination most conducive to pulsational instability (see, e.g., Simon and Stothers 1969). This is

$$
\beta=0.510, \quad \Gamma_{3}-1=0.372 .
$$

Furthermore, we may write

$$
\frac{1}{L} \frac{\partial}{\partial q}\left(\delta L_{r}\right)=f \frac{\partial l^{*}}{\partial q}+l^{*} \frac{\partial f}{\partial q},
$$

where, for a radiative star with constant opacity,

$$
l^{*}=4\left(x^{*}+t^{*}\right)+\frac{d t^{*}}{d \ln T} .
$$

The remaining unexplicit quantity in equation (6) is

$$
\begin{gathered}
\frac{\partial^{3} U}{\partial S \partial \rho^{2}}=\left.\frac{\partial}{\partial \rho}\right|_{S}\left[\frac{T}{\rho}\left(\Gamma_{3}-1\right)\right]=\left(\Gamma_{3}-1\right) \frac{T}{\rho^{2}}\left[\left(\Gamma_{3}-1\right)-1\right] \\
+\left.\frac{T}{\rho} \frac{\partial \beta}{\partial \rho}\right|_{S} \frac{\partial}{\partial \beta}\left(\Gamma_{3}-1\right) .
\end{gathered}
$$


With

we easily find

$$
1-\beta=\frac{a T^{4}}{3 P},
$$

$$
\left.\frac{\partial \beta}{\partial \rho}\right|_{S}=\frac{(1-\beta)}{\rho}\left[\Gamma_{1}-4\left(\Gamma_{3}-1\right)\right],
$$

and after some algebra

$$
\frac{\rho^{2}}{2 T} \frac{\partial^{3} U}{\partial S \partial \rho^{2}} \equiv W_{1}(\beta)=\frac{327 \beta^{3}-1128 \beta^{2}+1312 \beta-512}{9(8-7 \beta)^{3}} .
$$

The function $W_{1}(\beta)$ is always negative, with endpoint values of -0.111 , and a minimum of -0.149 at $\beta \simeq 0.93$. A glance at the last integral in equation (6) shows that this term serves to energize pulsations as long as the luminosity increases outward, and to damp pulsations as long as the luminosity decreases outward. For $\beta=0.510$, we have $W_{1}(\beta)=-0.119$.

We are now in a position to evaluate $L_{P}^{\prime} / L$. Results are displayed in Table 1 . It is easily seen that contributions arising from the additional term in $\delta(d S / d t)\left(I_{2}\right)$ and from the integral $J_{2}$ are approximaiely equal, and both contribute to energizing the pulsation.

\begin{tabular}{|c|c|c|c|c|}
\hline Model & $I_{1}$ & $I_{2}$ & $J_{2}$ & $L_{P}^{\prime} / L$ \\
\hline $\begin{array}{l}\text { Standard } \\
\text { model..... } \\
\text { White dwarf. }\end{array}$ & $\begin{array}{l}-4.8 \\
-18\end{array}$ & $\begin{array}{l}0.20 \\
1.6\end{array}$ & $\begin{array}{l}0.18 \\
1.3\end{array}$ & $\begin{array}{l}-4.4 \\
-15\end{array}$ \\
\hline
\end{tabular}

TABLE 1

VALUES OF THE STABILITY INTEGRALS

Their combined effect, however, is quite small, amounting to less than 8 percent of the ordinary damping.

\section{WHITE DWARF}

We shall discuss here the model of Marshak (1940) treated by Ledoux and SauvenierGoffin (1950). Though somewhat outdated, this model is quite adequate for our purposes. In particular, the luminosity distribution $f(q)$ is rather similar to that obtained from more modern treatments of cooling white dwarfs (e.g., Vila 1969).

Following Ledoux and Sauvenier-Goffin, we shall take

where

$$
z^{*}=-3 x^{*}=\text { const. }, \quad t^{*}=\left(\Gamma_{T}-1\right) z^{*},
$$

$$
\Gamma_{T}-1=\frac{x^{2}+2}{3\left(x^{2}+1\right)},
$$

and $x$ is the ratio of the Fermi momentum to $m c$.

We begin with the second integral in equation (6). Integrating by parts, we obtain

$$
I_{2}=4\left(x^{*}\right)^{2}-18\left(x^{*}\right)^{2} \int_{0}^{1}\left(\Gamma_{T}-1\right) f d\left(\Gamma_{T}-1\right),
$$

where we have taken $\Gamma_{T}-1=\frac{2}{3}$ at the stellar surface. Given the model parameters, we may easily evaluate the integral, obtaining $I_{2}=1.6\left(x^{*}\right)^{2}$. 
Turning now to $J_{2}$, we must calculate

$$
\begin{aligned}
\frac{\partial^{3} U}{\partial S \partial \rho^{2}} & =\left.\frac{\partial}{\partial \rho}\right|_{S}\left[\frac{T}{\rho}\left(\Gamma_{T}-1\right)\right] \\
& =\left(\Gamma_{T}-1\right) \frac{T}{\rho^{2}}\left[\left(\Gamma_{T}-1\right)-1\right]+\left.\frac{T}{\rho} \frac{\partial x}{\partial \rho}\right|_{S} \frac{\partial}{\partial x}\left(\Gamma_{T}-1\right) .
\end{aligned}
$$

Using the familiar expressions for a degenerate electron gas (Chandrasekhar 1939) and retaining only terms of lowest order in $\left(k T / m c^{2}\right)$, we find

and finally,

$$
\left.\frac{\partial x}{\partial \rho}\right|_{s}=\frac{x}{3 \rho}
$$

$$
\frac{\rho^{2}}{2 T} \frac{\partial^{3} U}{\partial S \partial \rho^{2}} \equiv W_{2}(x)=-\frac{2 x^{4}+7 x^{2}+2}{18\left(x^{2}+1\right)^{2}} .
$$

This function falls to a minimum of -0.153 at $x=1$. At the endpoints $(x=0, x \rightarrow \infty)$, $W_{2}(x)=-0.111$. Since $W_{2}(x)<0$, it turns out again that, for luminosity increasing outward, $J_{2}$ is an energizing term.

We have for the present case

$$
J_{2}=-9\left(x^{*}\right)^{2} \int_{0}^{1} W_{2}(x) d f .
$$

The values of $x$ for our model range from 2.4 at the center to 0.4 at the surface, encompassing values of $\left|W_{2}(x)\right|$ in the narrow range $0.13-0.15$. Using an average value $\left\{W_{2}(x) \mid=0.14\right.$, we obtain

$$
J_{2}=1.3\left(x^{*}\right)^{2}
$$

The remaining term in equation (6) is given by Ledoux and Sauvenier-Goffin:

$$
I_{1}=-18\left(x^{*}\right)^{2} \text {. }
$$

Setting $x^{*}=1$, we again summarize our results in Table 1 . In the present case, as with the standard model, the contributions $I_{2}=1.6$ and $J_{2}=1.3$ both serve to energize, and are roughly equal. Here, however, their effect is somewhat more important, offsetting about 16 percent of the ordinary damping.

\section{IONIZATION ZONES}

When ionization zones cover substantial regions of the stellar matter, the pulsational stability of a star can be strongly affected. In the absence of thermal imbalance, such effects have been studied in the literature in great detail.

When thermal imbalance exists in a star, the terms $I_{2}$ and $J_{2}$ begin to contribute, and they are affected by ionization through the thermodynamic coefficients $\left(\Gamma_{3}-1\right)$ and $\left(\rho^{2} / 2 T\right) \partial^{3} U / \partial S \partial \rho^{2}$. (It should be noted that the quasi-adiabatic theory may not be adequate here, depending upon the location of the ionization zones in a given star. The theory should be good enough, however, to give us some picture of the relative importance of thermal imbalance to the pulsational stability.)

If, in the presence of ionization zones, we impose the restriction that only one critical state of ionization exists in a given range of temperature and density, then we may write (Ledoux 1958)

$$
\Gamma_{1}=\frac{\left[16-12 \beta-1.5 \beta^{2}+\beta(4-1.5 \beta+\beta h) A_{T, P}\right]\left[1+A_{\rho, T}\right]}{12-10.5 \beta+\beta(1.5+h) A_{T, \rho}},
$$


where

$$
\Gamma_{3}-1=\frac{\Gamma_{1}-\beta\left(1+A_{\rho, T}\right)}{(4-3 \beta)+\beta A_{T, \rho}}
$$

$A_{\rho, T}=-\frac{B}{1+B}, \quad A_{T, \rho}=\frac{B}{1+B}(1.5+h), \quad A_{T, P}=B\left[2.5+h+\frac{4(1-\beta)}{\beta}\right]$.

Further, if $X$ represents the abundance (by number) of the element with critical ionization, $y$ the fraction of the electrons in question that have been removed, and $x$ the total number of free electrons per ion, then

$$
B=\frac{X x y(1-y)}{x(1+x)+X y(1-y)} .
$$

The quantity $h$ is the ratio of the ionization potential to $k T: h=\chi / k T$.

Once more, we must calculate the quantity

$$
\frac{\rho^{2}}{2 T} \frac{\partial^{3} U}{\partial S \partial \rho^{2}} \equiv W_{3}(\rho, T)=\frac{1}{2}\left(\Gamma_{3}-1\right)\left[\left(\Gamma_{3}-1\right)-1\right]+\left.\frac{1}{2} \frac{\partial}{\partial \ln \rho}\right|_{S}\left(\Gamma_{3}-1\right) .
$$

The necessary derivatives are $(\partial \beta / \partial \rho)_{S}$, given once more by equation ( 7$)$, and

$$
\left.\frac{\partial x}{\partial \rho}\right|_{S}=\frac{(1+x)}{\rho}\left[A_{\rho, T}+\left(\Gamma_{3}-1\right) A_{T, \rho}\right] .
$$

Finally, after cumbersome but straightforward computation we obtain

$$
\begin{aligned}
& \left.\frac{\partial}{\partial \ln \rho}\right|_{S}\left(\Gamma_{3}-1\right)=\llbracket(1-\beta)\left[\Gamma_{1}-4\left(\Gamma_{3}-1\right)\right]\left[6+A_{T, \rho}(6-4 h)\right] \\
& +\beta[6(1-\beta)-(4-3 \beta) h]\left\{\frac{Q(1+x) A_{T, \rho}}{(1+B)^{2}}\left[\left(\Gamma_{3}-1\right)(1.5+h)-1\right]+h\left(\Gamma_{3}-1\right) A_{\rho, T}\right\} \\
& +\beta\left(\Gamma_{3}-1\right) h A_{T, \rho}\left[4-3 \beta+\beta A_{T, \rho}\right] \rrbracket\left[12-10.5 \beta+\beta(1.5+h) A_{T, \rho}\right]^{-2},
\end{aligned}
$$

where

$$
Q=\frac{(1+x) x^{2}(1-2 y)+[X y(1-y)]^{2}-X x^{2} y(1-y)}{[x(1+x)+X y(1-y)]^{2}} .
$$

To get some idea of the size of $W_{3}(\rho, T)$, we have calculated it for a range of densities and temperatures for four different cases of critical ionization: $\mathrm{H} \mathrm{I}, \mathrm{He} \mathrm{I}, \mathrm{He}$ II, and C VI. The ratio of partition functions for the states involved was always taken to be the ratio of statistical weights. The latter quantities, along with values for ionization potentials, were taken from Unsöld (1955). In each case a composition consisting solely of the element in question was assumed for simplicity. This means, of course, that the effect of the ionization zones will be overestimated.

Table 2 presents these results. The last entry in the table is the ratio

$$
R=\frac{W_{3}(\rho, T)}{\left(\Gamma_{3}-1\right)^{2}}
$$

Since ordinary damping terms (at least for the case of radiative damping) as well as the integral $I_{2}$ will be proportional to $\left(\Gamma_{3}-1\right)^{2}$, the quantity $R$ gives some guide as to the importance of the last integral in equation (6).

In evaluating Table 2 , it will be useful to compare $W_{3}(\rho, T)$ with the quantities $W_{1}(\beta)$ and $W_{2}(x)$ calculated in previous sections. We first note that $W_{3}$ is not necessarily nega- 
TABLE 2

Some Thermodynamic Properties of Ionization Zones

\begin{tabular}{|c|c|c|c|c|c|c|c|c|c|c|c|c|c|c|}
\hline \multirow[b]{3}{*}{$\log T$} & \multicolumn{14}{|c|}{ H I, $x(e v)=13.59$} \\
\hline & \multicolumn{5}{|c|}{$\log \rho=-9.0$} & \multicolumn{6}{|c|}{$\log \rho=-5.0$} & \multicolumn{3}{|c|}{$\log \rho=-2.0$} \\
\hline & 3.7 & 3.8 & 4.0 & 4.1 & 4.2 & 3.9 & 4.0 & 4.1 & 4.4 & 4.5 & 4.6 & 4.2 & 4.6 & 5.3 \\
\hline$y$ & 0.000 & 0.005 & 0.523 & 0.956 & 0.997 & 0.001 & 0.008 & 0.045 & 0.792 & 0.943 & 0.984 & 0.006 & 0.219 & 0.945 \\
\hline$s$ & 0.996 & 0.992 & 0.980 & 0.970 & 0.943 & 1.000 & 1.000 & 1.000 & 1.000 & 1.000 & 1.000 & 1.000 & 1.000 & 1.000 \\
\hline$\Gamma_{3}-1$ & 0.620 & 0.319 & 0.086 & 0.239 & 0.525 & 0.597 & 0.407 & 0.231 & 0.261 & 0.449 & 0.602 & 0.544 & 0.372 & 0.648 \\
\hline$\left.\frac{\partial}{\partial \ln \rho}\right|_{S}\left(\Gamma_{3}-1\right)$ & -0.300 & -0.462 & +0.008 & +0.287 & +0.150 & -0.290 & -0.322 & -0.060 & +0.108 & +0.304 & +0.180 & -0.149 & +0.058 & +0.022 \\
\hline $\mathrm{w}_{3}(\rho, T)$ & -0.268 & -0.340 & -0.036 & +0.053 & -0.050 & -0.265 & -0.282 & -0.119 & -0.042 & +0.028 & -0.030 & -0.199 & -0.088 & -0.103 \\
\hline \multirow[t]{3}{*}{$\mathrm{R}$} & -0.70 & -3.33 & -4.80 & +0.92 & -0.18 & -0.74 & -1.70 & -2.23 & -0.62 & +0.14 & -0.08 & -0.67 & -0.64 & -0.24 \\
\hline & \multicolumn{14}{|c|}{ He I, $x(\mathrm{ev})=24.58$} \\
\hline & \multicolumn{5}{|c|}{$\log p=-8.0$} & \multicolumn{4}{|c|}{$\log \beta=-4.0$} & \multicolumn{5}{|c|}{$\log 0=-1.0$} \\
\hline $\log T$ & 4.0 & 4.1 & 4.2 & 4.3 & 4.5 & 4.2 & 4.5 & 4.7 & 4.8 & 4.4 & 4.6 & 4.8 & 5.0 & 5.5 \\
\hline$y$ & 0.002 & 0.036 & 0.357 & 0.924 & 1.000 & 0.004 & 0.479 & 0.962 & 0.991 & 0.005 & 0.061 & 0.283 & 0.646 & 0.979 \\
\hline 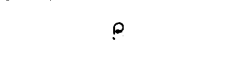 & 0.988 & 0.977 & 0.965 & 0.952 & 0.838 & 1.000 & 1.000 & 1.000 & 1.000 & 1.000 & 1.000 & 1.000 & $i .000$ & 1.000 \\
\hline$\Gamma_{1}$ & 1.465 & 1.150 & 1.099 & 1.192 & 1.527 & 1.463 & 1.230 & 1.508 & 1.631 & 1.547 & 1.401 & 1.407 & 1.505 & 1.663 \\
\hline$\Gamma_{3}^{-1}$ & 0.451 & 0.129 & 0.077 & 0.165 & 0.463 & 0.446 & 0.157 & 0.466 & 0.619 & 0.531 & 0.346 & 0.321 & 0.420 & 0.657 \\
\hline$\left.\frac{\partial}{\partial \ln \rho}\right|_{s}\left(\Gamma_{3}-1\right)$ & -0.692 & -0.054 & +0.003 & +0.126 & -0.025 & -0.416 & +0.026 & +0.367 & +0.161 & -0.194 & -0.022 & +0.056 & +0.112 & +0.013 \\
\hline $\mathrm{w}_{3}(0, \mathrm{~T})$ & -0.470 & -0.083 & -0.034 & -0.006 & -0.137 & -0.331 & -0.054 & +0.0 .59 & -0.038 & -0.222 & -0.124 & -0.081 & -0.066 & -0.106 \\
\hline
\end{tabular}




\begin{tabular}{|c|c|c|c|c|c|c|c|c|c|c|c|c|c|c|}
\hline \multirow[b]{3}{*}{$\log T$} & \multicolumn{14}{|c|}{ He II, $x(\mathrm{ev})=54.4$} \\
\hline & \multicolumn{5}{|c|}{$\log \mu=-8.0$} & \multicolumn{5}{|c|}{$\log \mu=-4.0$} & \multicolumn{4}{|c|}{$\log \rho=-1.0$} \\
\hline & 4.4 & 4.5 & 4.6 & 4.7 & 4.9 & 4.6 & 4.7 & 4.8 & 5.1 & 5.2 & 5.0 & 5.2 & 5.4 & 5.7 \\
\hline $\mathrm{y}$ & 0.000 & 0.019 & 0.524 & 0.969 & 1.000 & 0.000 & 0.006 & 0.096 & 0.961 & 0.990 & 0.009 & 0.143 & 0.520 & 0.896 \\
\hline$=$ & 0.912 & 0.840 & 0.766 & 0.659 & 0.329 & 1.000 & 1.000 & 1.000 & 0.999 & 0.998 & 1.000 & 1.000 & 1.000 & 1.000 \\
\hline$\Gamma_{1}$ & 1.565 & 1.237 & 1.041 & 1.353 & 1.391 & 1.657 & 1.515 & 1.278 & 1.563 & 1.642 & 1.600 & 1.488 & 1.542 & 1.648 \\
\hline$\Gamma_{3}^{-1}$ & 0.516 & 0.247 & 0.120 & 0.331 & 0.353 & 0.657 & 0.497 & 0.219 & 0.532 & 0.632 & 0.584 & 0.421 & 0.467 & 0.625 \\
\hline$\left.\frac{\partial}{\partial \ln \rho}\right|_{S}\left(\Gamma_{3}-1\right)$ & -0.102 & -0.366 & +0.004 & +0.215 & -0.001 & -0.090 & -0.591 & -0.051 & +0.314 & +0.096 & -0.164 & +0.017 & +0.104 & +0.053 \\
\hline $\mathrm{w}_{3}(0, T)$ & -0.176 & -0.276 & -0.051 & -0.003 & -0.115 & -0.158 & -0.421 & -0.111 & +0.033 & -0.068 & -0.203 & -0.114 & -0.072 & -0.090 \\
\hline R & -0.66 & -4.54 & -3.53 & -0.03 & -0.92 & -0.37 & -1.70 & -2.31 & +0.11 & -0.17 & -0.60 & -0.64 & -0.33 & -0.23 \\
\hline
\end{tabular}

C VI, $x(\mathrm{ev})=489.84$

\begin{tabular}{|c|c|c|c|c|c|c|c|c|c|c|c|c|c|c|}
\hline \multirow{3}{*}{$\log T$} & \multicolumn{14}{|c|}{ C VI, $x(\mathrm{ev})=489.84$} \\
\hline & \multicolumn{4}{|c|}{$\log 0=-7.0$} & \multicolumn{5}{|c|}{$\log \beta=-3.0$} & \multicolumn{5}{|c|}{$\log \hat{\beta}=0.0$} \\
\hline & 5.4 & 5.5 & 5.6 & 5.7 & 5.6 & 5.7 & 5.8 & 5.9 & 6.0 & 5.8 & 6.0 & 6.2 & 6.4 & 6.7 \\
\hline$y$ & 0.002 & 0.206 & 0.928 & 0.997 & 0.002 & 0.039 & 0.357 & 0.822 & 0.965 & 0.001 & 0.032 & 0.334 & 0.776 & 0.967 \\
\hline$=$ & 0.094 & 0.051 & 0.029 & 0.015 & 0.996 & 0.992 & 0.986 & 0.974 & 0.950 & 1.000 & 1.000 & 1.000 & 0.999 & 0.993 \\
\hline$\left.\frac{\partial}{\partial \ln p}\right|_{s}\left(-z_{3}-1\right)$ & -0.003 & -0.026 & +0.007 & 0.000 & -0.182 & -0.433 & +0.020 & +0.270 & +0.113 & -0.019 & -0.126 & +0.056 & +0.101 & -0.006 \\
\hline $\mathrm{w}_{3}(\mu, T)$ & -0.113 & -0.122 & -0.108 & -0.111 & -0.207 & -0.339 & -0.086 & +0.017 & -0.068 & -0.122 & -0.184 & -0.097 & -0.071 & -0.118 \\
\hline $\mathrm{R}$ & -1.00 & -1.17 & -0.97 & -1.00 & -0.52 & -1.87 & -1.29 & +0.12 & -0.25 & -0.28 & -0.53 & -0.40 & -0.20 & -0.28 \\
\hline
\end{tabular}


tive. In certain regions, the derivative $\left[\partial\left(\Gamma_{3}-1\right) / \partial \ln \rho\right]_{S}$ can become positive and large enough to outweigh the first term in equation (8). This happens in general for large positive $Q$ and for $\beta \simeq 1$. In that case, a star with luminosity increasing outward would tend to be damped (and one with luminosity decreasing outward, to be energized) by the last integral in equation (6). However, this effect seems to occur in a relatively small part of the $(\rho, T)$-plane. Overall, those regions with $W_{3}<0$ are likely to dominate, giving a result qualitatively the same as in the absence of ionization zones.

Perhaps a more important difference involves the size of the effect. We see from Table 2 that $W_{3}$ can become quite large in absolute value, exceeding the maximum values of $\left|W_{1}\right|$ and $\left|W_{2}\right|$ by a factor as great as 3 . Furthermore, the ratio $R$ attains large negative values (e.g., for $\mathrm{He}_{\mathrm{I}}, \log \rho=-8, \log T=4.2$ ), particularly in low density regions. The largest values occur for $\Gamma_{3}-1 \lesssim 0.1$, and run as high as $R=-5.78$ for the cases calculated. (As one goes to ionizations with larger potentials, e.g., $\mathrm{C}$ vI, the effect begins to diminish. This is due to increasing domination by radiation pressure in the low-density regions, and to the fact that the ratio $x$ of electrons to ions is increasing. In fact, as $\beta \rightarrow 0$ or $x \rightarrow \infty, W_{3} \rightarrow W_{1}$.) The corresponding ratios for $W_{1}$ and $W_{2}$ can never exceed unity in absolute value, and are generally much smaller.

Thus it turns out that the thermodynamics of ionization zones is such that the contribution to stability analysis of "thermal-imbalance" terms may become greatly enhanced. Whether this actually happens depends, of course, on the detailed properties of the stars in question. We shall have more to say about this in the next section.

\section{DISCUSSION}

Up to now we have seen that the thermal-imbalance terms investigated will, in general, tend to energize pulsations in stars with luminosity increasing outward. This will be the case for gravitationally contracting stars or for stars experiencing thermal cooling. Expansion, on the other hand, will tend to damp pulsations.

In $\$$ III and IV it has been shown for two different cases that the extra terms make a small contribution compared with that from ordinary damping terms. It is simple and instructive to compare these extra terms further with the energizing due to nuclear reactions. For the case of the white dwarf, Ledoux and Sauvenier-Goffin (1950) have shown that nuclear energizing would be enough to overcome the damping for very modest values of the temperature exponent $\nu(\nu=9.5$ for a core source; $\nu=2.6$ for a shell source). Thus, this energizing is at least 6 times as effective as the thermal-imbalance contributicn given in Table 1 .

For the standard model, a comparison is not so straightforward. However, since, apart from the temperature exponent, the amount of nuclear energizing will depend mainly on the size of pulsational amplitudes in the burning region (let us say in the core) it seems reasonable to compare the standard model with a model having a similar value of relative radius amplitude at its center. One such model in the literature is that of a 28.2 $M \odot$ main-sequence star (Schwarzschild and Härm 1958). It has a central radius amplitude $x^{*}{ }_{c}=0.39$, while the value for standard model of $\S$ III is $x^{*}{ }_{c}=0.32$. With a temperature exponent $\nu=13$, Schwarzschild and Härm (1959) found nuclear energizing $L_{P N} / L-4.4-$ a value more than an order of magnitude larger than that due to thermal imbalance in the standard model (Table 1).

The above comparisons are, of course, somewhat artificial, since (1) any region of a star in nuclear thermal equilibrium $\left(d S_{1} / d t \approx 0\right)$ cannot contribute to the thermal-imbalance terms and (2) the standard model could not represent in detail a star with physically reasonable nuclear processes. Nevertheless, we may safely say that, given the thermodynamics of $\$ \S I I I$ and IV, ordinary pulsation terms will tend greatly to outweigh thermal-imbalance terms, with the latter providing only relatively small corrections.

For the thermodynamics of $\S \mathrm{V}$ (ionization zones) the outlook changes somewhat. 
We have seen in this case that the coefficient $\left|W_{3}\right|$ can become relatively large, tending to enhance the contribution due to thermal imbalance. On the other hand, such effects occur mainly in diffuse regions, where the density is low. The classic example is in the ordinary Cepheid variables. In the well-known modern calculations of Cepheid variability (Christy 1966a; Baker and Kippenhahn 1965), thermal imbalance is ignored, and the equilibrium luminosity taken as constant in the energizing region. Because of the extreme central condensation of Cepheid models, only the outer 1 or 2 percent of the stellar mass is affected by the pulsation (Christy 1966b), and even if thermal imbalance does exist in these layers, it is hardly to be expected that they contribute enough luminosity to affect the stability significantly.

Further, it seems possible on the basis of our results to rule out important contributions for any star expanding or contracting across the $\mathrm{H}-\mathrm{R}$ diagram during normal post-main-sequence evolution, at least through core helium burning. Although considerable regions of such stars can be in a state of thermal imbalance (see, e.g., Iben 1966), the central condensation of these objects $\left(\rho_{c} /\langle\rho\rangle \gtrsim 10^{3}-10^{5}\right.$, as compared with $\rho_{c} /\langle\rho\rangle=54$ for the standard model) is such that radiative damping will almost certainly crush any of the extra terms we have considered.

Similarly, cooling degenerate bodies such as white dwarfs are highly unlikely to become pulsationally unstable as a result of contributions from thermal imbalance, such contributions being simply too small to overcome damping in the thin radiative zones at the surface of these stars.

VII. AREAS FOR FUTURE WORK

We begin this section by noting that our investigation remains incomplete because of the omission of the last integral in equation (1). However, there are reasons to believe that inclusion of this term will not change qualitatively the conclusions of the previous section. Calling the integral in question $\mathrm{LK}_{2}$, taking the time average, and normalizing with the luminosity, we obtain

$$
K_{2}=-\int_{0}^{1}\left(\Gamma_{3}-1\right) \frac{d f}{d q}\left(\frac{\delta \rho}{\rho}\right)_{2} d q
$$

where $(\delta \rho / \rho)_{2}$ indicates the surviving time-averaged second-order amplitudes.

We have already seen in the cases studied that the integrals $I_{2}$ and $J_{2}$ provide small, approximately equal contributions. Since we must expect that $(\delta \rho / \rho)_{2} \approx\left(z^{*}\right)^{2}$, it follows that $K_{2} \approx I_{2}$ or $J_{2}$, or perhaps is somewhat larger due to the relative largeness of $\left(\Gamma_{3}-1\right)$ compared with the thermodynamic coefficients of $I_{2}$ and $J_{2}$. Even if the integral $K_{2}$ proved to have the same sign as $I_{2}$ and $J_{2}$ in the cases investigated, it would need to be nearly 10 times larger for the white dwarf and more than 20 times larger than $I_{2}$ or $J_{2}$ for the standard model in order to qualitatively affect the stability. Although a full second-order theory must be developed to evaluate the size of $K_{2}$ exactly, such large values seem unlikely.

On the other hand, when the terms $I_{2}$ and $J_{2}$ begin to become comparable to the ordinary damping or energizing terms, the integral $K_{2}$ must obviously be considered in detail. We shall suggest two stages of stellar evolution for which this may be the case:

1. Thermally unstable shell-burning stars.-Rose has found that certain thermally unstable models become pulsationally unstable as well, for both helium (Rose 1967) and hydrogen (Rose 1968) shell burning. The seat of the instability is strongly enhanced nuclear energizing arising from a thermal runaway in the shell. However, the flood of photons released in the shell is almost totally absorbed in the expanding layers above, with the luminosity dropping by orders of magnitude from shell to surface. Although Rose has not published the runs of luminosity or pulsational amplitudes, he does give enough information to enable us to put limits on $I_{2}$ and $J_{2}$ for his unstable models. 
Table 3 shows the relevant quantities for two typical models, 3B (Rose 1967) and 2A (Rose 1968). The entries are, in order, the shell and surface luminosities, the relative pressure amplitudes $p^{*} \equiv \delta P / P$, the nuclear energizing $N_{1}$ (normalized by $L$ ), and the ordinary damping $I_{1}$. The last two entries give limits on the thermal-imbalance terms. The following paragraphs show how these limits were calculated.

Since the envelopes of Rose's models are either nondegenerate or only slightly degenerate, we have used $\left(\Gamma_{3}-1\right)^{2}$ and $W_{1}(\beta)$ as the thermodynamic coefficients for $I_{2}$ and $J_{2}$, respectively, and have set $\beta=1$ for simplicity. Using these quantities and neglecting $f_{\text {surface }}$ with respect to $f_{\text {shell }}$, we obtain

$$
I_{2}+J_{2}=-\frac{1}{5} f_{\text {shel1 }}\left\langle\left(p^{*}\right)^{2}\right\rangle,
$$

where $\left\langle\left(p^{*}\right)^{2}\right\rangle$ is an average over the envelope luminosity distribution, and $f_{\mathrm{shell}}=$ $L_{N} / L$.

The sum (9) is negative (i.e., a damping term), as we expect for luminosity decreasing outward. Using the limiting values of $\left(p^{*}\right)^{2}$ as given by Rose, we obtain the last two entries in Table 3.

TABLE 3

PUlsational Characteristics For TWo THERMALly UNSTABLE MODELS: 3B (Rose 1967) AND 2A

(Rose 1968)

\begin{tabular}{|c|c|c|}
\hline Variable & 3B & $2 \mathrm{~A}$ \\
\hline $\begin{array}{l}L_{N} / L \odot \ldots \ldots \ldots \\
L / L \odot \ldots \ldots \ldots \\
p^{*}(\text { surface }) \ldots \\
p^{*}(\text { shell }) \ldots \ldots \\
N_{1} \ldots \ldots \ldots \ldots \\
I_{1} \ldots \ldots \ldots \ldots \\
\left(I_{2}+J_{2}\right)_{\min } \ldots \ldots \\
\left(I_{2}+J_{2}\right)_{\max } \ldots \ldots\end{array}$ & $\begin{aligned} & 1.5 \times 10^{6} \\
- & 110 \\
= & 0.29 \\
- & 3.5 \times 10^{3} \\
- & 4.5 \\
- & 2.4 \times 10^{2} \\
- & 4.0 \times 10^{5}\end{aligned}$ & $\begin{aligned} & 2 \times 10^{4} \\
& 200 \\
- & 15 \\
- & 0.50 \\
& 50 \\
- & 6.3 \\
- & 5.0 \\
- & 4.5 \times 10^{3}\end{aligned}$ \\
\hline
\end{tabular}

Consider the minimum damping due to thermal imbalance $\left(I_{2}+J_{2}\right)_{\min }$. For model $2 \mathrm{~A}$ it is comparable to the ordinary damping $I_{1}$, while for $3 \mathrm{~B}$ it far exceeds $I_{1}$. In both cases the nuclear energizing dominates, so there is no qualitative effect. On the other hand, if we use the upper limit $\left(I_{2}+J_{2}\right)_{\max }$, the thermal-imbalance damping completely overwhelms all other terms and the instability is nullified. Because pulsational amplitudes will tend to drop off rapidly from the surface inward, the true value of $I_{2}+J_{2}$ is probably closer to the lower limit than to the upper limit. However, the size of even the minimum terms indicate that thermal-imbalance contributions must be taken into account in any evaluation of the pulsational stability during a thermal runaway. To do this will require a full second-order theory.

2. Stars in pre-main-sequence contraction.- Here instability is favored by three characteristics: (1) a large percentage of the luminosity is provided by gravitational contraction; (2) the central condensation is lowered substantially by the influence of convection; and (3) deep ionization zones can exist in such stars, depending on mass and the state of evolution. Detailed models of pre-main-sequence contraction have been constructed by Iben (1965) and Ezer and Cameron (1967).

A start on a stability analysis for such stars has been made by Kato and Unno (1967) and Okamoto (1967). The former authors developed a second-order theory and, with the aid of a number of restrictive assumptions, managed to write the stability integrals 
(1) in approximate form. This form was in turn used by Okamoto to test the stability of a fully convective homologously contracting polytrope of index 1.5 . Ionization zones were neglected.

Emphasizing the tentative nature of the calculation, Okamoto concluded that stars with masses $<2 M \odot$ were probably pulsationally unstable against energization due to thermal imbalance. Establishment of this conclusion must await the development of a full second-order theory including all the terms in equation (1).

The author gratefully acknowledges support of an NRC postdoctoral research associateship under the National Aeronautics and Space Administration. I would also like to thank Dr. R. Stothers for helpful comments and criticism, and a referee for a useful suggestion.

\section{REFERENCES}

Baker, N., and Kippenhahn, R. 1965, Ap. J., 142, 868.

British Association for the Advancement of Science. 1932, Mathematical Tables, Vol. 2.

Chandrasekhar, S. 1939, An Introduction to the Study of Stellar Structure (Chicago: University of Chicago Press).

Christy, R. F. $1966 a, A$ p. J., 144, 108.

. 1966b, Ann. Rev. Astr. and Ap., 4, 353.

Eddington, A. S. 1959, The Internal Constitution of the Stars (New York: Dover Publications).

Ezer, D., and Cameron, A. G. W. 1967, Canadian J. Phys., 45, 3429.

Iben, I., Jr. 1965, Ap.J., 141, 993.

1966, ibid., 143, 516.

Kato, S., and Unno, W. 1967, Pub. Astr. Soc. Japan, 19, 1.

Ledoux, P. 1958, Hdb. d. Phys., 51, 605.

Ledoux, P., and Sauvenier-Goffin, E. 1950, Ap. J., 111, 611.

Marshak, R. E. 1940, Ap. J., 92, 321.

Okamoto, I. 1967, Pub. Astr. Soc. Japan, 19, 384.

Rose, W. 1967, Ap. J., 150, 193.

. 1968, ibid., 152, 245.

Schwarzschild, M. 1941, Ap. J., 94, 245.

Schwarzschild, M., and Härm, R. 1958, Ap. J., 128, 348. . 1959, ibid., 129, 637.

Simon, N. R., and Stothers, R. 1969, Ap. J., 156, 377.

Thomas, L. H. 1930, M.N.R.A.S., 91, $122,619$.

Unsöld, A. 1955, Physik der Sternatmosphären (Berlin: Springer-Verlag).

Vila, S. 1969 (unpublished models). 